\title{
Fokus Starke Lernbeziehungen
}

\section{Ein Schulversuch}

DOI: https://doi.org/10.53349/sv.2021.i3.a126

\begin{abstract}
Der Schulversuch „Fokus Starke Lernbeziehungen“ (FSL) verfolgt das Ziel, die Zahl der an einer Klasse tätigen Lehrpersonen zu reduzieren und damit die Lehr- und Lernbeziehungen zu stärken. Der integrative Unterricht soll weiterentwickelt und die Schulorganisation vereinfacht werden. Dazu werden Mittel aus Unterstützungs- und Fördermassnahmen zugunsten vermehrten Teamteachings von zwei Regelklassenlehrpersonen umgelagert. Die Lehrpersonen unterrichten möglichst alle Fächer und verfolgen dabei auch die Ziele von Deutsch als Zweitsprache (DaZ), der Integrativen Förderung (IF) und der Begabtenförderung. Die schulischen Heilpädagog*innen sowie die DaZ-Lehrpersonen beraten und unterstützen sie dabei. Zwölf der dreizehn Versuchsschulen nutzen die Möglichkeit, FSL bis auf Weiteres weiterzuführen, weil sie vom Schulmodell überzeugt sind. Für ihr langjähriges Engagement und die Beiträge aus der Alltagspraxis zu diesem Artikel danke ich den Schulleitungen an dieser Stelle herzlich.
\end{abstract}

Schul- und Unterrichtsentwicklung, Lernorientierte Zusammenarbeit, Integrative Förderung, Wissenstransfer, Schulversuch

\section{Ausgangslage}

Die Bildungsdirektion Kanton Zürich startete aufgrund der vom Schulfeld - seit Längerem beklagten belastenden Arbeitssituation 2009 das Projekt „Belastung - Entlastung im Schulfeld“. Mittels einer differenzierten Analyse der Belastungssituationen an der Volksschule und unter Einbezug aller wichtigen Stakeholder wurden verschiedene Entlastungsmöglichkeiten erarbeitet. Einer dieser Vorschläge lautete, die Anzahl der Lehrpersonen pro Klasse zu verringern, weil deren grosse Anzahl an einer Klasse zu erhöhter Belastung der Schüler*innen (SuS) sowie der Lehrpersonen selber führe. Der Schulversuch Fokus Starke Lernbeziehungen (FSL) setzt hier an.

Ein Schulversuch legitimiert die beteiligten Schulen, von den regulären Organisationsstrukturen, Unterrichtsformen und -inhalten projektartig abzuweichen und diese im Hinblick auf ei- 


\section{\# schuleverantworten}

führungskultur_innovation_autonomie

ne Übernahme für das breite Schulfeld zu prüfen. Idealerweise wird ein Schulversuch engmaschig begleitet, reflektiert und überprüft. Der Gesetzgeber erhält damit Grundlagen, die es erlauben, gegebenenfalls die Neuerungen flächendeckend einzuführen oder Elemente davon weiterzuentwickeln und dem breiten Schulfeld zur Verfügung zu stellen.

FSL ist ein Schulentwicklungsprojekt, welches die Schulorganisation vereinfachen, einen Beitrag zur Weiterentwicklung des integrativen Unterrichts ${ }^{1}$ und zur Entlastung der Regelklassenlehrpersonen leisten soll. Die mit FSL verbundenen Zielsetzungen betreffen sowohl die Schulorganisation als auch die Unterrichtsentwicklung. Damit geht eine Befähigung der Lehrund Fachpersonen für die im Schulversuch an sie gestellten Anforderungen einher. Die Überprüfung des Projektes FSL erfolgte zwischen 2014 und 2017 mittels Daten aus Beobachtungen und Interviews. Der Evaluationsbericht von Ende $2017^{2}$ gibt einen detaillierten Einblick in die nach vier Jahren mit dem Projekt erreichte Wirksamkeit der anvisierten Ziele. Die Ergebnisse der externen Evaluation zeigen folgende Resultate:

- FSL vereinfacht die Schulorganisation,

- leistet einen Beitrag zur Weiterentwicklung des integrativen Unterrichts,

- ermöglicht eine effizientere Zusammenarbeit,

- stellt einen Gewinn für die Lehrpersonen dar.

- Allerdings: Die Stärkung der Lernbeziehungen kann nicht nachgewiesen werden. ${ }^{3}$

\section{Rahmenvorgaben}

FSL ermöglicht den teilnehmenden Schulen sich so zu organisieren, dass grundsätzlich zwei Lehrpersonen pro Klasse alle Fächer unterrichten und dabei auch die Ziele der Integrativen Förderung (IF), des Unterrichts in Deutsch als Zweitsprache (DaZ) und der Begabtenförderung (BG) verfolgen.

Dafür werden die für individuelle Unterstützungs- und Fördermassnahmen zur Verfügung stehenden Mittel zugunsten der Regelklasse zusammengefasst. Sie kommen den Klassen durch vermehrtes Teamteaching (TT) und in Form von Beratung der Klassenlehrpersonen durch Fachpersonen für IF, DaZ und BG zugute. Ein kleineres, mit zusätzlichen Ressourcen verstärktes Klassenteam erhält damit bessere Voraussetzungen, um beständige und tragfähige Lehr- und Lern-Beziehungen zu den Schüler*innen aufzubauen. Wesentliche Elemente für die Erreichung der im Projekt anvisierten Wirkungsziele lassen sich wie folgt aufgliedern:

\section{Weniger Bezugspersonen im Klassenzimmer}

- Weniger Bezugspersonen führen zu konstanteren Beziehungen, welche die Kinder stärken.

- Besondere Bedürfnisse und Fähigkeiten im Rahmen des Regelunterrichts lassen sich fokussierter berücksichtigen. 


\section{\# schuleverantworten}

führungskultur_innovation_autonomie

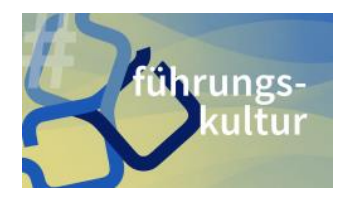

- Sprachliche Kompetenzen, insbesondere von Kindern, deren Muttersprache nicht Deutsch ist, oder aus sozial benachteiligten Familien können kontinuierlich gefördert werden.

\section{Stärkung der Regelklassenlehrpersonen}

- Entlastung durch Reduktion von Absprachen und Koordinationsaufgaben.

- Erweiterung der methodischen und fachlichen Kompetenzen der Lehrpersonen.

- Stärkung des Berufsbildes der Lehrpersonen ${ }^{4}$, die im Regelunterricht auch für Massnahmen besonderer pädagogischer Bedürfnisse zuständig sind.

\section{Vereinfachung der Schulorganisation}

- Weniger Koordinationsaufwand zwischen Fachlehrpersonen (z.B. für IF, DaZ) und Klassenlehrpersonen und Stärkung der pädagogisch-methodischen Unterrichtsentwicklung. Zudem soll der Schulversuch wesentliche Erkenntnisse zur Weiterentwicklung des Schulmodells sowie pädagogische, strukturelle, rechtliche und finanzielle Grundlagen für die Entscheidungsfindung am Ende des Schulversuchs erwirken.

\section{Die Versuchsschulen und ihre Motivation}

Von Beginn weg wurden drei Staffeln geplant. Bei der Auswahl der Versuchsschulen wurde auf einen für den Kanton Zürich repräsentativen Mix aus städtischen, ländlichen und Schulen im städtischen Umland mit unterschiedlichen sozio-ökonomischen Merkmalen geachtet (vgl. Anhang). Der Versuchsstart mit der ersten Staffel im Schuljahr 2013/14 begann mit fünf Schuleinheiten. Eine davon enthielt zwei Kindergärten einer Primarschule. Das Jahr darauf, in der zweiten Staffel, kamen vier weitere Schuleinheiten hinzu (eine davon bildeten alle Kindergärten der Gemeinde Kloten ${ }^{5}$ ) und im folgenden Schuljahr, 2015/16, begann die dritte Staffel mit drei weiteren Versuchsschulen. Im Schuljahr 2018/19 wurden auf Gesuch der Schulbehörde hin die restlichen drei Primarschulen der Gemeinde Kloten, deren Kindergärten bereits im Schulversuch integriert waren, als eigenständige Schuleinheiten mit ihren jeweiligen Kindergärten aufgenommen. Die Gemeinde Kloten hatte aufgrund eigener Evaluationen das Modell FSL für ihre Primarschulen als sehr geeignet eingestuft.

Die Möglichkeit, den Förderunterricht auf weniger Personen zu verteilen und die damit gewonnenen Ressourcen integrativ mittels TT an die Schüler*innen weiterzugeben, war für die Versuchsschulen ein attraktiver Ansatz für eine verstärkte integrative Förderung in den Bereichen Sprache, IF und BG. Insbesondere im Kindergarten waren die Herausforderungen für eine alleinige Lehrperson oftmals kaum mehr zu bewältigen. In Schulen wie Kloten, Rümlang, Schlieren oder Wetzikon und Winterthur sprechen viele Kinder zu Beginn der obligatorischen Schulzeit ihre Herkunftssprache und wenig bis gar kein Deutsch. Zudem sind die Vorstellungen, wie Selbstständigkeit zuhause gefördert werden kann, kulturell sehr unterschiedlich. Die 


\section{\# schuleverantworten}

führungskultur_innovation_autonomie

Schule leistet hier einen wichtigen Beitrag zur Integration und zum Schulerfolg. Die meisten Versuchsschulen setzen deshalb im Kindergarten einen grossen Teil der zusätzlichen Ressourcen ein, um den heterogenen Voraussetzungen besser zu begegnen und die Schüler*innen frühzeitig zu einer erfolgreichen Lernbereitschaft hinzuführen.

\section{Herausforderungen für die Schulführung}

\subsection{Ressourcenplanung}

Der eigens für FSL zur Planung der Mittel entwickelte Ressourcenplaner (RP) ${ }^{6}$ basiert auf den kantonalen, den Gemeinden gesetzlich zustehenden Ressourcen. Je nach Schule kommen zusätzliche kommunale Ressourcen, beispielsweise aus Therapie, Aufgabenhilfe, Assistenzen etc., hinzu. Nach Abzug der Ressourcen für die Beratung durch IF- und DaZ-Fachpersonen werden die verbleibenden Ressourcen den einzelnen Klassen als TT-Lektionen zugewiesen. Zudem sind aus dem RP Anzahl Klassen, Schüler*innen und Lehrpersonen (inklusive Ausbildungsportfolio) pro Klasse ersichtlich.

\subsection{Zusammenstellung der Klassentandems}

Eine der grossen Herausforderungen für die Schulleitungen ist die personelle Zusammenstellung der einzelnen Klassentandems. Die beiden an einer Klasse tätigen Lehrpersonen müssen idealerweise zusammen alle Fächer abdecken. Sie sind gemeinsam für die Förderung ihrer Schüler*innen in einem integrativen Unterrichtssetting verantwortlich und werden dabei von den Beratungsfachpersonen für IF und DaZ unterstützt. Beim regelmässigen Austausch zwischen Schulleitung und Lehrpersonen über ihre Zusammenarbeit im Tandem setzen die Schulen ein Formular ${ }^{7}$ ein, das im Rahmen des Schulversuchs dafür entwickelt wurde. Das Projekt unterstützt die Schulen diesbezüglich zudem mit Coaching- und Supervisionsangeboten.

Für alle Leitungen der Versuchsschulen ist die Bildung der Klassentandems, die den Klassenunterricht gemeinsam verantworten, die Basis für die integrative Förderung. Das TT nährt sich aus den gebündelten Ressourcen für IF, DaZ und BG, was in den TT-Lektionen wirksam sein soll. In Kloten beispielsweise bewährt es sich sehr, die Klassenteams in gelingende Zusammenarbeit einzuführen, indem mit einer sorgfältig ausgefüllten und regelmässig zu überarbeitenden Zusammenarbeitsvereinbarung sowie mittels gezieltem Coaching der Klassenteams gearbeitet wird. Auch andere Schulleitungen wie Rifferswil betonen die Wichtigkeit der Zusammenarbeitsvereinbarung für die Entwicklung des Tandems und des Unterrichts. Das Angebot für Coaching haben in der Versuchsphase alle Schulen genutzt. Nach Aussagen der Schulleitungen haben sie zu gut etablierten Klassenteams geführt oder waren hilfreich für die Auftrennung und Neubildung von Teams. 


\subsection{Zuteilung von Ressourcen}

Durch die Ressourcenbündelung stehen, je nach Grösse der Schule und Anteil Kinder nicht deutscher Muttersprache, pro Klasse zwischen 120 und 160 Stellenprozente zur Verfügung. Es liegt in der Kompetenz der Schulleitung, aus den ermittelten Ressourcen den IF- und DaZFachpersonen Beratungsstunden und den Klassen TT-Lektionen zuzuweisen. Mehrheitlich teilen die Schulen den Kindergärten eine ansehnliche Menge an TT-Lektionen zu. Die Erfahrung zeigt, dass sich ein Einsatz vermehrter Ressourcen bei der IF und der Sprachförderung im Kindergarten später beim Eintritt in die Primarstufe auszahlt. Auf der Primarstufe können verschiedene Kriterien für eine Zuweisung von mehr oder weniger TT-Lektionen an die Klasse ausschlaggebend sein: Anzahl der Schüler*innen mit individuellem Förderbedarf (auch BG), Anzahl der DaZ- Schüler*innen, Berufserfahrung der Lehrpersonen, Klassengrösse etc.

Für die Schulleitungen liegt die Schwierigkeit vor allem in einer „gerechten“ Verteilung der zur Verfügung stehenden Ressourcen. Allen ist bewusst: „Mit einer transparenten Kommunikation der angewendeten Zuteilungskriterien lassen sich Konflikte im Team proaktiv vermeiden" Die von den Schulleitungen meistgenannten und angewendeten Kriterien für die Verteilung der Ressourcen sind:

- Anzahl an IF- und DaZ- Schüler*innen sowie der Schüler*innen mit besonderem Förderbedarf in den einzelnen Klassen,

- Berufserfahrung der Lehrpersonen insbesondere im Bereich Classroom Management, Unterrichtsplanung und Lehrplanerfüllung,

- Balance halten zwischen ausreichender und nötiger Anzahl Beratungsstunden für IF und DaZ und der dafür vorhandenen fachlichen Personalressourcen,

- Bereitschaft der Lehrpersonen zur Entwicklung der Zusammenarbeit im Tandem, aber auch interdisziplinär mit den Fachpersonen für SHP und DaZ.

\subsection{Unterrichtsentwicklung}

Nach dem Organisationsaufbau orteten die Versuchsschulen sehr bald Bedarf an zusätzlichem, fachlichem und methodischem Wissen im Unterrichtsbereich, insbesondere bezüglich TT sowie individueller und sprachlicher Lernförderung. Den Versuchsschulen standen finanzielle Mittel für spezifische Weiterbildungsangebote zur Verfügung. In Zusammenarbeit mit Ausbildungsinstitutionen konnten innen massgeschneiderte Bildungsangebote als Schulinterne Weiterbildung (SCHILW) sowie für Gruppen, Klassen oder Einzelpersonen gemacht werden. Es standen Weiterbildungen in den Bereichen Förderung (Förderplanung, -diagnostik und Begabtenförderung), Sprache (Sprachstanderfassung, Integrativer DaZ-Unterricht), Unterricht (Binnendifferenzierung, Individualisierung, Lerncoaching, Lernbeziehungen, Leistungsbeurteilung), Zusammenarbeit (TT und TT-Prozesse, Effektives TT) und Beratung (Coach to the Coaches [sic], Teamcoaching) zur Verfügung. Begleitend und parallel dazu wurden die 
nachfolgend kurz beschriebenen spezifischen Grundlagen und Instrumente für die Unterrichtsentwicklung und die Zusammenarbeit in FSL entwickelt. Die Fertigstellung des Fachkonzepts Integrierte Sprachförderung auf der Kindergarten- und Primarstufe ${ }^{8}$ im Oktober 2017 fiel zusammen mit der Einführung des Lehrplans $21^{9} \mathrm{im}$ Schuljahr 2018/19 und ermöglichte den Versuchsschulen eine bestmögliche Vernetzung ihrer Bemühungen im Bereich der Sprachförderung. Der Wunsch nach Praxisbeispielen für die Gestaltung lernwirksamer TTLektionen führte zur Erstellung der Fachbroschüre Lernorientierte Zusammenarbeit ${ }^{10}$. Sie enthält Beispiele aus dem 1. und 2. Zyklus mit dem Fokus Sprachförderung.

\subsection{Lernorientierte Zusammenarbeit}

In Zusammenarbeit mit der Pädagogischen Hochschule Zürich (PHZH), dem Institut Unterstrass (IU) und unter der Führung der Fachhochschule Nordwestschweiz (FHNW) konnte ein praxisbezogenes Projekt zur Ermittlung der Potenziale von Zusammenarbeit im Team bezüglich Individueller Förderung und Verstärkung der Sprachförderung durchgeführt werden. Vier Tandems aus verschiedenen Versuchsschulen und Zyklen wurden während der Planung einer ihrer TT-Lektionen von ihrer Beratungsperson und den Fachpersonen aus der Projektgruppe beobachtet. Im Anschluss fand eine gemeinsame Besprechung der konkret beobachteten Unterrichtsplanung des Tandems statt. Danach setzten die Lehrpersonen die Lektion unter Berücksichtigung der aus der Besprechung gewonnenen Erkenntnisse um, wiederum unter Beobachtung. Das daraus entstandene Filmmaterial und die Beobachtungsprotokolle bildeten zusammen mit dem theoretischen Know-how der Fachpersonen die Grundlage der Fachbroschüre Lernorientierte Zusammenarbeit (s.o.). Darin finden sich beispielhaft Unterrichtssequenzen aus der Praxis. Diese sind sprachtheoretisch und methodisch-didaktisch eingebettet, erläuternd und ansprechend beschrieben. Aus diesen Bausteinen ist auch das modulare Weiterbildungskonzept Lernorientierte Zusammenarbeit entstanden. ${ }^{11}$

Aus Sicht der Versuchsschulen ist die Lernbegleitung und die Lernorientierte Zusammenarbeit (TT) ein gewinnbringender Ansatz. Im Laufe der Jahre kann die Zusammenarbeit optimiert und an die Erfordernisse der Klassenzusammensetzung angepasst werden. Die Erfahrungen in den Versuchsjahren haben jedoch gezeigt, dass die jeweiligen Wechsel der Lehrpersonen im Tandem jedes Mal eine grosse Herausforderung darstellen. „Der Grundgedanke von FSL muss immer wieder aufgefrischt werden, ansonsten ,verkommt' FSL zu, einfach mehr Team-Teaching' und die gezielte Förderung bleibt auf der Strecke“ (Neftenbach). „TT und Lernorientierte Zusammenarbeit sind anspruchsvoll. Viele meiner Tandems kamen frisch von der Ausbildung; sie sind gut im Austauschen und arbeiten auf gleicher Augenhöhe miteinander. Es ist aber ihre erste Stelle; Classroom Management und Lehrplanerfüllung stehen an erster Stelle. TT heisst dann: eine Lehrperson führt und die andere unterstützt. Im zweiten Jahr kann dann der Fokus vermehrt auf das gemeinsame Planen, Scaffolding und Umsetzen gelegt werden. Es braucht Zeit und Unterstützung, aber v.a. auch Zeit, die Schritte auszuprobieren." (Kloten) 


\subsection{Entwicklung der Beratungsfunktion}

Ein Knackpunkt zeigte sich in der Funktion der Beratungspersonen. Das Projekt ging von der Annahme aus, dass Fachlehrpersonen für Schulische Heilpädagogik und DaZ beraterische Grundkenntnisse für ihre Tätigkeit an den Schulen mitbringen. Die Funktion Beratung für IF, DaZ und BG an FSL-Schulen musste jedoch von Beginn weg erarbeitet werden. Der erwartete Wissenstransfer zwischen Fachpersonen und Lehrpersonen konnte nicht in allen Versuchsschulen in gleichem Masse hergestellt werden. Spezifische Kurse insbesondere zu den Themen Zusammenarbeit, Coaching sowie die Teilnahme an Supervisionen ermöglichten den Fachpersonen, fachlich-methodisches Know-how für ihre Tätigkeit zu erwerben. Diese Angebote wurden von Fach- wie Klassenlehrpersonen sehr geschätzt. In Zusammenarbeit mit den FSL-Schulleitungen, Vertretungen der PHZH, dem IU sowie der Höheren Fachschule für Heilpädagogik $(\mathrm{HfH})$ entstand eine Beschreibung der Funktion Beratung in FSL-Schulen ${ }^{12}$. Damit wurde eine wichtige Grundlage für die weitere, schulspezifische Entwicklung der Funktion an den Versuchsschulen geschaffen. Eine im Auftrag des Volksschulamtes von der $\mathrm{HfH}$ erstellte Expertise $^{13}$ zur Beratung leistet einen wichtigen Beitrag, um das Ausmass und die Tragweite der Funktion zu definieren, und zeigt anhand von einzelnen Beispielen Umsetzungsformen. „Die Expertise konnte aufzeigen, wie bedeutsam Beratung im System ist und hat wesentlich dazu beigetragen, sich mit Gelingensfaktoren von gutem Coaching auseinanderzusetzen. Ganz konkret, indem ein regelmässiger Austausch zwischen den Beratungspersonen mit den Schulleitungen stattfindet, welcher zu wichtigen Weiterbildungsimpulsen für die gesamte Schulentwicklung führt. Das System Schule rückt damit in den Fokus der gezielten und lösungsorientierten Schulentwicklung, welche auf Kooperationen beruht. Der Transfer von Expertenwissen ins System Schule wirkt zunehmend. Fachliche Beratung zieht Kreise bis hin zu den anderen Fachpersonen im Bereich Sonderpädagogik (Therapien wie Logopädie und Psychomotorik), die sich ergänzend mit den FSL-Beratungspersonen für eine gemeinsame Schulentwicklung engagieren" (Kloten).

Die Praxis zeigt, dass die Beratungsfunktion in FSL ein Entwicklungsprozess war und immer noch ist. „Das „Beratungs-Know-how“ kommt immer noch sehr unterschiedlich bei den Klassenlehrpersonen an. Die Frage der Akzeptanz und der Stufenzugehörigkeit spielt oft auch eine wichtige Rolle, wenn beispielsweise eine SHP aus dem Kindergarten Lehrpersonen aus Zyklus II beraten soll. Es gibt Schulen mit je einer Beratungsperson für IF oder DaZ. Die Lehrpersonen haben damit zwei Beratungspersonen zur Seite. Einzelne Schulen haben heute je eine Beratungsperson für Zyklus I und II, die sowohl für IF als auch für DaZ und BG zuständig ist. Es ist wichtig, immer wieder die Rollen und Erwartungen zu klären. Die Beratungsperson macht die Triage und klärt, was in ihren Kompetenzen liegt. Die Förderung bleibt in der Verantwortung der Lehrpersonen." (Kloten) 


\section{Folgen der Evaluation}

Im Frühjahr 2018 entschied die Bildungsdirektion Kanton Zürich aufgrund der Evaluationsergebnisse, das Modell FSL im Kanton nicht flächendeckend einzuführen. Entscheidend war dabei auch, dass die Analyse der Daten aus der Erhebungsphase zwischen Mai 2014 und Juni 2017 keinen Leistungszuwachs bezüglich Lernbeziehung nachwies.

In der Zwischenzeit war der Schulversuch für die teilnehmenden Schulen bis Ende Schuljahr 2021/22 verlängert worden. Die Versuchsschulen erhielten damit Planungssicherheit bis zum Entscheid, ob sie auch im Regelbetrieb als FSL-Schulen weitergeführt werden können. Die Bildungsdirektion wünschte in dieser Zeit vertieft abzuklären, welche Elemente des Schulversuchs sich unter welchen Bedingungen günstig auf die Regelklassenlehrpersonen auswirken und gleichzeitig bessere Leistungen der Schülerinnen und Schüler gewährleisten. Zudem wurde ein Einfliessen des Expertenwissens der DaZ- und IF-Lehrpersonen in den Unterricht angestrebt. Die im Schulversuch gewonnenen Erkenntnisse sollten dann in geeigneter Form als Impuls für die Schul- und Unterrichtsentwicklung der Volksschulen des Kantons Zürich zur Verfügung gestellt werden.

Anfangs 2021 wurde von der Bildungsdirektion Kanton Zürich das Projekt „ME flex“ initiiert mit dem Ziel, dass Gemeinden die Mittel, welche den Schulen zur Verfügung stehen, zielgerichtet und wirkungsvoll einsetzen können. Dazu soll der Handlungsspielraum in der Organisation der schulischen Angebote erweitert werden. Die Versuchsschulen erhielten daraufhin die Möglichkeit, sich bis Ende Schuljahr 2020/21 zu entscheiden, ob sie bis auf Weiteres eine FSL-Schule bleiben wollten oder einen Rückbau in die bestehende Organisationsform wünschten. Von den dreizehn Versuchsschulen haben sich zwölf für eine Fortsetzung entschieden.

Dass die Datenanalyse der Evaluation keinen Leistungszuwachs bezüglich Lernbeziehung nachweisen konnte, erstaunte einige Schuleiter*innen aus den Versuchsschulen. Gerade diesbezüglich hatten sie gegensätzliche Eindrücke erhalten. Die Evaluationsresultate forderten sie heraus, genauer hinzuschauen, ob und wo sie sich geirrt haben mochten. Dies gab der Unterrichtsentwicklung einen neuen Schub. Die Schulleitungen achteten bei der Wahl von Weiterbildungen vermehrt auf nachhaltige, modulare Angebote mit Reflexionsanteilen. Mehrere Versuchsschulen wählten beispielsweise Weiterbildungen, die sich vertieft mit dem Verhältnis von $T T$ und Unterrichtsplanung oder Formen von $\pi$ für die gezielte Förderung praxisbezogen auseinandersetzten.

\section{Hinweise und Erkenntnisse aus FSL für Führungspersonen}

Nachfolgend wird stellvertretend das Fazit, das die Schulen Kloten, Neftenbach und Rifferswil zum Schulversuch FSL ziehen, wiedergegeben: 


\section{\# schuleverantworten}

führungskultur_innovation_autonomie

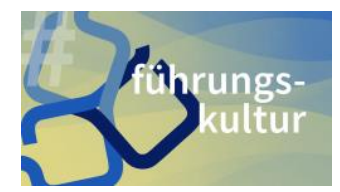

- Die Führung eines Schulversuchs ist insbesondere bei grossen Schulen sehr herausfordernd. Es gilt, das Feuer für innovative Schulprojekte bei interessierten Lehrpersonen zu entfachen und über einen längeren Zeitraum warm zu halten. Es gibt begabte Lehrpersonen, welche vom Projekt einer inklusiven Pädagogik und Didaktik überzeugt sind und die, zusammen mit einer mutigen Schulleitung, eine Mehrheit des Teams mit gezielter pädagogischer Schulentwicklung voranbringen können. Es braucht auf Führungsebene dazu Zeit, Kontinuität und v.a. auch die Lust und Neugier für neue didaktische Unterrichtsmodelle. Die Aufgabe der Schulleitung verändert sich durch FSL auch insofern, als neue Formen der Zusammenarbeit entstehen und neue didaktische Modelle zum Einsatz kommen. (Kloten)

- Die Individuelle Lernbegleitung bleibt, insbesondere bei Junglehrpersonen, die sehr mit dem Klassenmanagement und der allgemeinen Pädagogik beschäftigt sind, eine grosse Herausforderung. Mit der Broschüre „Lernorientierte Zusammenarbeit“ wurde ein konkreter Praxisbezug geschaffen. Die Checkliste ${ }^{14}$ dazu bietet sehr gute Orientierung, wie Integrative Sprachförderung als Team geplant werden kann. Das Know-how, wie gut zusammengearbeitet werden kann, ist generell gewachsen, bleibt aber nach wie vor ein Erfolgs- oder Misslingensfaktor. (Kloten, Neftenbach)

- Die Lehrpersonen schätzen die Zusammenarbeit. Die Leitung muss jedoch zwingend ein Auge auf der Förderung haben; diese muss gezielt angegangen und darf nicht beliebig werden. (Neftenbach)

- FSL hat wirklich Schulentwicklung ausgelöst und gute neue Konzepte hervorgebracht. Wenn die Tandems spielen, dann entsteht ein „FSL Mehrwert“ für unsereSchüler*innen. Entwicklungsarbeit braucht viel Zeit und konstante FSL-Tandems. Als Schulleiterin stärke und unterstütze ich diese Tandems und sorge für Rahmenbedingungen, die ermöglichen, dass sich die Lehrpersonen auf FSL einlassen können. (Rifferswil)

\section{Resümee}

Der Evaluationsbericht attestiert dem Schulversuch FSL, vier der fünf anvisierten Ziele erreicht zu haben (vgl. Kapitel 5 und Anmerkung 2). Für die Lehrpersonen brachte er einen für sie messbaren Gewinn, der sich in der oft gehörten Aussage „Ich bin nicht alleine“ widerspiegelt. Die Weiterentwicklung des integrativen Unterrichts fand vor allem in der Zeit nach dem Vorliegen der Evaluationsresultate ab 2018 statt. Hier zeigt sich einmal mehr, dass es für Schul- und Unterrichtsentwicklungsprojekte einen langen Atem braucht. In den letzten Jahren wurde der Fokus vermehrt auf die Zusammenarbeit in den Tandems und auf die verstärkte Förderung in den gemeinsam erteilten Lektionen gesetzt (vgl. Anmerkungen 8 und 10-14). Dies begann sich für die Schulleitungen und die Lehrpersonen positiv auf den Unterricht auszuwirken (vgl. Kapitel 5 und obige Aussagen der Schulleitungen). Die Lehr- und Fachpersonen wurden durch die Auseinandersetzungen, den Austausch im Team und an entsprechenden Weiterbildungen für die multiprofessionelle Zusammenarbeit sensibilisiert. Insbesondere die IF- und die DaZ-Beratungen durch die Fachpersonen begannen an Bedeutung und Professionalität zu gewinnen. Sie wurden von den Schulleitungen aktiver unterstützt und von den Lehrpersonen vemehrt für methodisch-didaktische Unterstützung in Fragen der individuellen 
Förderung beigezogen. Die Stärkung der Lernbeziehungen konnte die Evaluation in der kurzen Beobachtungszeit zwar nicht nachweisen. Aber gerade dieser Umstand spornte anschliessend einige Schulleitungen und Lehrpersonen der Versuchsschulen an. Sie führte zum genaueren Hinsehen und zu den Fragen: „Was macht eine gute Lernbegleitung aus? Welche Kompetenzen und Rahmenbedingungen braucht es dafür?" Der Schulversuch FSL hat erfolgreiche und nachhaltige Schul- und Unterrichtsentwicklungsimpulse ermöglicht.

\section{Anmerkungen}

${ }^{1}$ Eine Übersicht zur Praxis der integrativen Förderung im Kanton Zürich findet sich in der handreichung_integrative_und_individualisierte_lernfoerderung.pdf

${ }^{2}$ Der Evaluationsbericht findet sich unter www.zh.ch/unterrichtsentwicklung $\rightarrow$ FSL $\rightarrow$ Evaluation Fokus Starke Lernbeziehungen.

${ }^{3}$ Folgen der Evaluation: vgl. Kapitel 5.

${ }^{4}$ Grundsätzlich sind die Lehrpersonen der Regelklassen - bis zu einem gewissen Grad (vgl. Anmerkung 1) auch für die individuelle und sprachliche Förderung sowohl leistungsstarker wie -schwacher Schüler*innen zuständig (,[...] die im Regelunterricht auch für Massnahmen besonderer pädagogischer Bedürfnisse zuständig sind. "). Leider ist das Delegieren dieser Aufgaben an Fachpersonen für Daz und IF oft die erste Lösung.

${ }^{5}$ Die FSL-Schulen bestehen aus Primarschuleinheiten mit Klassen aus Kindergarten sowie aus dem 1. und 2. Zyklus. In Kloten wurden in einem ersten Anlauf nur die Kindergartenklassen aus verschiedenen Schuleinheiten in den Schulversuch aufgenommen. Später wurden alle Primarschuleinheiten Klotens aufgenommen und die Kindergarteneinheit wurde aufgelöst. Die Kindergärten waren vor dem Versuchsstart bereits organisatorisch einer bestehenden Schuleinheit zugewiesen. Somit verblieben sie in FSL.

${ }^{6}$ Dokumente zur Ressourcenplanung: www.zh.ch/unterrichtsentwicklung $\rightarrow$ FSL $\rightarrow$ Downloads für die Versuchsschulen $\rightarrow$ Ressourcenplanung und Anleitung Ressourcenplanung.

${ }^{7}$ Zusammenarbeitsvereinbarung für Klassenteams: www.zh.ch/unterrichtsentwicklung $\rightarrow$ FSL $\rightarrow$ Downloads für die Versuchsschulen $\rightarrow$ Mustervorlage Zusammenarbeitsvereinbarung für Klassenteams.

${ }^{8}$ Fachkonzept integrierte Sprachförderung: www.zh.ch/unterrichtsentwicklung $\rightarrow$ FSL $\rightarrow$ Fachkonzept integrierte Sprachförderung auf der Kindergarten- und Primarstufe.

${ }^{9}$ Gesamtschweizerische Informationen zum LP 21 sind unter www.lehrplan21.ch zu finden, zum LP 21 des Kantons Zürich finden sich Informationen unter Lehrplan 21.

${ }^{10}$ Fachbroschüre Lernorientierte Zusammenarbeit: www.zh.ch/unterrichtsentwicklung $\rightarrow$ FSL $\rightarrow$ Fachbroschüre Lernorientierte Zusammenarbeit. Beispiele aus dem 1. und 2. Zyklus mit dem Fokus Sprachförderung.

${ }^{11}$ Ein modulares WB-Angebot zu Lernorientierter Zusammenarbeit startet ab 2022 und wird von der Fachhochschule Nordwestschweiz (FHNW) vorerst als SCHILW (Schulinterne Weiterbildung) mit einem Schulungsumfang von total drei halben Tagen angeboten.

${ }^{12}$ Aufgabenbeschrieb der Beratungsfunktion: www.zh.ch/unterrichtsentwicklung $\rightarrow \mathrm{FSL} \rightarrow$ Downloads für die Versuchsschulen $\rightarrow$ Beratungsfunktion Aufgabenbeschrieb.

${ }^{13}$ Expertise zur Beratungsfunktion: www.zh.ch/unterrichtsentwicklung $\rightarrow \mathrm{FSL} \rightarrow$ Expertise zur Beratungsfunktion.

${ }^{14}$ Checkliste zur Zusammenarbeit im Team: www.zh.ch/unterrichtsentwicklung $\rightarrow$ FSL $\rightarrow$ Checkliste „Zusammenarbeit im Team zur Sprachförderung". 


\section{Anhang}

FSL Versuchsschulen seit Schuljahr 2013/14 bis 2021/22

\begin{tabular}{|c|c|c|c|}
\hline $\begin{array}{l}\text { 1. Staffel } \\
\text { PS* Rifferswil } \\
\text { PS Letten, Zürich-Waidberg } \\
\text { PS Hofacker, Schlieren } \\
\text { PS Guldisloo, Wetzikon } \\
\text { Kindergarten Marthalen } \\
\text { (bis SJ 2019/20) } \\
\text { * Primarschule }\end{array}$ & $\begin{array}{l}\text { 2. Staffel } \\
\text { PS Heerenweg/Aesch, Neftenbach } \\
\text { PS Worbiger, Rümlang } \\
\text { PS Lind, Winterthur-Altstadt } \\
\text { Kindergarten Kloten } \\
\text { (bis SJ 2018/19) }\end{array}$ & $\begin{array}{l}\text { 3. Staffel } \\
\text { PS Dorf/Feld, Kloten } \\
\text { PS Am Wasser, Zürich Waidberg } \\
\text { PS Ruggenacher 1, Regensdorf } \\
\text { (bis SJ 2021/22) }\end{array}$ & $\begin{array}{l}\text { Erweiterung Kloten } \\
\text { (ab SJ 2019/20) } \\
\text { PS Hinterwiden } \\
\text { PS Spitz } \\
\text { PS Nägelimoos }\end{array}$ \\
\hline
\end{tabular}

\section{Autorin}

Julia-C. Sanz, , MSc.

Wirtschafts- und Sozialgeographin, Projektleiterin FSL, Volksschulamt, Bildungsdirektion Kannton Zürich. Primarschullehrerin, Berufs-, Studien- und Laufbahnberaterin, Bildungsevaluatorin, Themenschwerpunkte: Frauen und Migration, Kulturkonflikte, Schul- und Unterrichtsentwicklung

Kontakt: julia.sanz@vsa.zh.ch 